\title{
Stem Cell Expansion and Fate Decision on Liquid Substrates Are Regulated by Self-Assembled Nanosheets
}

\author{
Dexu Kong ${ }^{1,2}$, Lihui Peng ${ }^{1,2}$, Stefania Di Cio ${ }^{1,2}$, Pavel Novak ${ }^{1,2}$ and Julien E. Gautrot ${ }^{1,2 *}$ \\ ${ }^{1}$ Institute of Bioengineering and ${ }^{2}$ School of Engineering and Materials Science, Queen Mary, \\ University of London, Mile End Road, London, E1 4NS, UK. \\ *Corresponding author.j.gautrot@qmul.ac.uk
}

\begin{abstract}
The culture of adherent cells is overwhelmingly relying on the use of solid substrates to support cell adhesion. Indeed, it is typically thought that relatively strong bulk mechanical properties (bulk moduli in the range of $\mathrm{kPa}$ to $\mathrm{GPa}$ ) are essential to promote cell adhesion and, in turn, regulate cell expansion and fate decision. In this report, we show that adherent stem cells such as mesenchymal stem cells and primary keratinocytes can be cultured at the surface of liquid substrates and that this phenomenon is mediated by the assembly of polymer nanosheets at the liquid-liquid interface. We use interfacial rheology to quantify this assembly and demonstrate the strong mechanical properties of such nanosheets. Importantly, we show that cell adhesion to such quasi-2D materials is mediated by the classical integrin/acto-myosin machinery, despite the absence of bulk mechanical properties of the underlying liquid substrate. Finally, we show that stem cell proliferation and fate decision are also regulated by the mechanical properties of these self-assembled protein nanosheets. Liquid substrates offer attractive features for the culture of adherent cells and stem cells and the development of novel stem cell technologies, as liquid-liquid systems are particularly well-adapted to automated parallel processing and scale up.
\end{abstract}

\section{Keywords}

Nanosheets; Interfacial Mechanics; Self-Assembly; Stem Cells; Cell Adhesion. 
Solid substrates displaying bulk moduli in the range of $\mathrm{kPa}$ (soft hydrogels and elastomers) to $\mathrm{GPa}$ (tissue culture polystyrene - TPS - or hydroxyapatite) are typically used for the culture of adherent cells and stem cells and as scaffolds for tissue engineering. Indeed, a wide range of cell phenotypes, such as cycling, ${ }^{1}$ cell spreading and motility, ${ }^{2-4}$ apoptosis, ${ }^{5}$ stem cell self-renewal ${ }^{6}$ and differentiation ${ }^{7}$, ${ }^{8}$ have been shown to correlate with the bulk mechanical properties of biomaterials. Although tissue culture plastic and glass are overwhelmingly used to expand stem cells, for the study of molecular biology and tissue engineering, such solid substrates present important drawbacks, in particular for the scale up of cell expansion (e.g in 3D bioreactors) and the design of parallel culture systems. In contrast to the culture of non-adherent cells that has been scaled up to large tanks, for the culture of algae, bacteria and eukaryotic cells such as $\mathrm{CHO}$ cells, for the production of chemicals, antibodies and therapeutics, ${ }^{9-11}$ the culture of adherent stem cells remains poorly scalable. Alternative strategies bypassing the use of solid substrates are therefore attractive.

A number of recent reports have highlighted that cell phenotype does not necessarily correlate with bulk mechanical properties of biomaterials used as extra-cellular matrix. Hence, the adhesion and differentiation of mesenchymal stem cells (MSCs) and human primary keratinocytes (HPKs) were not affected by the bulk mechanical properties of Sylgard 184 PDMS substrates. ${ }^{12}$ Similarly, MSCs cultured in soft 3D hydrogels based on degradable hyaluronic acid matrices committed towards osteogenic lineages, whereas those seeded in stiff matrices differentiated into adipocytes, ${ }^{13}$ the opposite trend to what was observed on 2D poly(acrylamide) hydrogels ${ }^{7}$ and within non-degradable 3D hydrogels. ${ }^{14}$ This was proposed to result from the ability of cells to degrade soft hydrogels sufficiently fast and spread, whereas stiffer hydrogels imposed a rounded shape to cells. In addition, local viscoelastic deformation was proposed to alter and regulate cell adhesion, modulating the molecular clutch mechanism previously proposed to account for cell sensing of matrix stiffness and resulting in changes in the fate of MSCs. ${ }^{15,16}$ At the molecular scale, single molecule force probe microscopy indicates that cells sense mechanical properties of their environment by exerting $<200 \mathrm{~nm}$ deformations on the matrix, ${ }^{17,} 18$ confirming the importance of local mechanics on cellular sensing. Therefore, the local 
mechanical and geometrical environment to which cells are exposed may dominate other bulk properties, and potentially evolves during cell culture as cells remodel their extra-cellular environment. ${ }^{19}$

Surprisingly, considering the importance of substrate mechanics on cell phenotype, cell culture at the surface of low viscosity liquids has been observed, mainly in the context of fibroblasts ${ }^{20-22}$ and myoblasts. ${ }^{23}$ This phenomenon has been studied with fluorinated oils, such as the fluorinated ether Novec 7500, and poly(dimethyl siloxane). In such systems, the use of surfactant molecules was reported to play an important role in the regulation of cell proliferation, although the underlying mechanism was not known. ${ }^{22}$ Recently, we presented evidence for the formation of mechanically strong protein nanosheets self-assembling at the interfaces between cell culture medium and the supporting oil. ${ }^{24,25}$ We observed a direct correlation between the concentration of the pro-surfactant pentafluorobenzoyl chloride (PFBC) and the interfacial shear modulus of interfaces to which bovine serum albumin adsorbed. In addition, this correlated with a gradual increase in the proliferation of the epidermal cell line HaCaT. The use of liquids for the culture of adherent cells and stem cells is attractive as it would bypass the need for cell detachment via enzymatic treatment for harvesting and transfer and would allow the simple handling of cell culture, for example using emulsion based culture systems (e.g. for 3D bioreactor). A main hurdle to develop such technologies is to promote the adhesion of stem cells to liquid-liquid interfaces via extra-cellular matrix (ECM) proteins.

In this report, we demonstrate the self-assembly of strong polyelectrolyte nanosheets based on poly(L-lysine) (PLL), promoting the adsorption of the ECM protein fibronectin and stem cell adhesion. Using interfacial rheology, we characterise the in situ assembly of PLL nanosheets and demonstrate the strong interfacial shear properties of the corresponding interfaces. We characterise the mechanism via which adherent stem cells such as HPKs and MSCs adhere to PLL nanosheet interfaces, after fibronectin adsorption. We demonstrate that these interfaces promote stem cell expansion at liquid-liquid interfaces, at comparable rates to those observed on solid substrates, and that the 
nanoscale mechanics of these interfaces regulates cell fate. Finally, we demonstrate the proof-ofconcept of using emulsions for stem cell expansion and their simple transfer to other substrates.

Our previous reports highlighted that proteins deposited from cell culture medium containing serum (of which albumin is a major component) and direct adsorption of bovine serum albumin from single protein solutions at the surface of Novec 7500 and PDMS oils allowed the formation of mechanically strong protein nanosheets able to support the growth of HaCaT cells. ${ }^{25}$ However, such interfaces did not allow the growth of adherent stem cells such as HPKs and MSCs (results not shown). We hypothesised that this lack of growth was the result of the poor adhesion of adherent stem cells to these interfaces and that the deposition of ECM proteins, which plays an essential role in the regulation of fate decision, ${ }^{26,27}$ was essential to enable stem cell proliferation. In order to promote ECM protein adsorption (such as fibronectin) to glass substrates, poly(L-lysine) (PLL) is typically deposited first. Therefore, we first studied the adsorption of PLL at oil-water interfaces using interfacial rheology, a methodology that is particularly sensitive to the adsorption of proteins and nanoparticles at liquid-liquid interfaces (Fig. 1). ${ }^{28-30}$ Owing to the modest amphiphilicity of PLL, we used the pro-surfactant pentafluorobenzoyl chloride (PFBC) to promote the assembly of PLL.

Upon addition of a PLL solution the interfacial shear modulus of liquid-liquid interfaces increased by nearly 4 orders of magnitude (Fig. 1A). This process was particularly affected by the $\mathrm{pH}$ of the PLL solution as strengthening of liquid-liquid interfaces was observed at $\mathrm{pH} 10.5$, compared to $\mathrm{pH} 7.4,8.5$ and 9.5 (corresponding interfaces displayed interfacial shear moduli 2 orders of magnitude lower, Fig. $1 \mathrm{~B}$ and $\mathrm{C})$. The interfacial shear moduli of interfaces generated at low (7.4) $\mathrm{pH}$ was also more strongly dependent on frequency than at high $\mathrm{pH}$ (10.5), indicating a more viscoelastic profile, and clearly indicated failure at lower frequencies. This was consistent with our observation that the stability of emulsions from PLL aqueous solutions and PFBC solutions in Novec 7500 oil (2/1 ratio) was excellent at $\mathrm{pH}$ 10.5, whereas emulsions destabilised in seconds at pH 7.4. Interestingly, the onset of the increase in interfacial modulus was delayed at pH 9.5, 8.5 and 7.4 (Supplementary Fig. 1), but the 
ultimate densities of PLL adsorbed (measured via fluorescence microscopy, with tagged PLL) were relatively comparable at different $\mathrm{pH}$. This suggests that, although PLL assembly is not prevented at low $\mathrm{pH}$, this polymer does not assemble into rigid structures. Overall, our data is consistent with PLL assembly at liquid-liquid interfaces mediated by the pro-surfactant PFBC at high $\mathrm{pH}$, resulting in considerable strengthening of the corresponding interfaces.

Albumin self-assembly at similar oil-water interfaces resulted in the formation of protein nanosheets with dry thicknesses in the range of $15-20 \mathrm{~nm} \cdot{ }^{25}$ To study whether PLL also formed nanosheets at liquid-liquid interfaces, we used scanning electron microscopy (Fig. 2A). PLL was assembled at the surface of oil droplets at high $\mathrm{pH}$, prior to thorough washing of the aqueous phase and drying of the oil in air (Novec 7500 has a boiling point of $128^{\circ} \mathrm{C}$ at 1 Bar). SEM images gave clear evidence of nanosheets with wrinkled morphologies comparable to those observed for albumin nanosheets. ${ }^{25}$ Characterisation of PLL nanosheets by atomic force microscopy also indicated comparable thicknesses (14 $\pm 2 \mathrm{~nm}$, Fig. 2B), consistent with the assembly of polymer monolayers. Finally, XPS analysis of the resulting PLL nanosheets revealed substantial levels of PFBC (see Fig. $2 \mathrm{C}$, corresponding to $13 \%$ functionalisation, based on atom \% and the structure represented in Fig. 1D), indicating the covalent coupling of this pro-surfactant to PLL. Overall, we propose that PLL assembly at fluorinated oil interfaces into polymer nanosheets is mediated by reaction with the pro-surfactant PFBC, through the formation of a fluorinated amide side group that pins PLL domains at the fluorinated oil interface (Fig. 1D). This results in the considerable strengthening of the corresponding interfaces, despite the quasi 2D-character of PLL nanosheets formed.

The adhesion of HPKs at the surface of PLL nanosheets was investigated next. Keratinocytes were seeded at oil-water interfaces at which PLL had been assembled at low or high $\mathrm{pH}$, followed by fibronectin coating, and their spreading was compared to that of cells adhering to TPS coated with fibronectin (Fig. 3A and B; slightly higher fibronectin adsorption to PLL nanosheets were observed, compared to TPS, Supplementary Fig. 2). Keratinocytes adhering to stiff PLL nanosheets (formed at 
high $\mathrm{pH}$ ) displayed comparable spreading to those adhering to TPS, significantly higher than those adhering to weak interfaces (generated at low $\mathrm{pH}$ ). To test the role of acto-myosin contractility in cell adhesion to liquid substrates, we treated cells with blebbistatin, the ROCK inhibitor Y27632 and the actin depolymerising drug Cytochalasin D (Fig. 3C). Treatment with blebbistatin and the ROCK inhibitor Y27632 led to a pronounced change in cell shape and a disruption of the actin cytoskeleton, despite no marked change in overall spreading, on both solid substrates and at oil interfaces. Cytochalasin D had a more pronounced impact on cell spreading, in addition to the disruption of the actin cytoskeleton, on solid as well as oil interfaces, confirming the importance of the actin cytoskeleton in cell adhesion at liquid-liquid interfaces. In addition, cell spreading dynamics was affected by the mechanical properties of the PLL interfaces formed. For this experiment, HaCaT cells were allowed to adhere at oil interfaces with soft or stiff PLL nanosheets for different periods of time prior to fixation and staining with phalloidin (Supplementary Fig. 3A). In this context, we observed again more extensive and faster spreading on TPS and stiff PLL nanosheets compared to the weaker oil-water interfaces, although HaCaT spreading was more extensive on TPS. Hence our results indicate that cell spreading and cytoskeleton organisation are regulated by the mechanical properties of liquid-liquid interfaces.

We next examined whether cell spreading on oil substrates was regulated by integrin adhesion, as is the case on solid substrates. Spreading was impaired by blocking integrin $\beta_{1}$ ligation using the antibody P5D2, ${ }^{31}$ both in the context of HPKs and HaCaT cells (HPKs, Fig. 3D and E; HaCaT cells, Supplementary Fig. 3B and C), indicating the importance of integrin $\beta_{1}$ to promote cell spreading on oils. In agreement with this behaviour, we found that HPKs spreading on stiff PLL nanosheets formed focal adhesions (vinculin staining in Fig 3F; talin, paxillin and phosphotyrosine in Supplementary Fig. 4) and assembled a structured actin cytoskeleton comparable to that of cells adhering to TPS (in the case of keratinocytes, no significant difference in fibre length, width or density, between cell spreading on stiff oil interfaces and TPS, Supplementary Fig. 5A and B). Cell spreading was also impaired when fibronectin was diluted with BSA (Supplementary Fig. 5 C and D). However, MSC spreading was 
unaffected by coating of PLL nanosheets with vitronectin instead of fibronectin. In comparison, cells adhering to weak liquid-liquid interfaces did not assemble focal adhesions and did not display any sign of actin cytoskeleton assembly. This was further confirmed by scanning ion conductance microscopy, a non-contact scanning probe microscopy technique particularly adapted to the imaging of very soft substrates, $^{32}$ which demonstrated the formation of lamellipodia and filopodia at the surface of stiff, but not soft oil interfaces (Fig. 3G). Hence cells sense the nanoscale mechanics of liquid interfaces via an integrin-mediated mechanism, relying on the assembly of focal adhesions and a mature actin cytoskeleton.

To establish the relevance of liquid substrates for stem cell expansion, we examined the impact of liquid interfaces on stem cell fate. Bulk mechanical properties often correlate with stem cell differentiation, ${ }^{33}$ however a few reports had indicated that stem cell differentiation is not necessarily regulated by bulk mechanical properties. ${ }^{13,16,34}$ We tested whether cell adhesion to low-viscosity liquids displaying no bulk mechanical strength would alter stem cell fate. In FAD medium and in nondifferentiating medium (KSFM), primary keratinocytes cultured on oils did not express the cornified envelop marker involucrin (below $15 \%$, Fig. 4A and B). In contrast, cells cultured on TPS or on oil functionalised with the protein resistant PLL-PEG remained rounded and differentiated to high levels (above $60 \%$ ), similarly to the suspension induced differentiation of keratinocytes. ${ }^{35}$ Overall, our results demonstrate that PLL nanosheets are sufficiently strong to allow cell spreading and that the lack of bulk mechanical strength did not affect keratinocyte fate decision significantly.

To demonstrate the versatility of liquid substrates for stem cell expansion, we cultured mesenchymal stem cells at the surface of Novec 7500 supplemented with the pro-surfactant PFBC and observed comparable proliferation rates to those measured on TPS (Fig. 4C). We confirmed that MSCs cultured in such conditions formed focal adhesions and assembled a structured actin cytoskeleton, as on TPS (Fig. 4D). Therefore, our results indicate that liquid substrates functionalised with polymer or protein 
nanosheets displaying strong mechanical properties and presenting ligands for integrin adhesion would be expected to promote stem cell spreading and proliferation.

Liquid substrates are therefore very general and versatile platforms for stem cell expansion and have direct implications for stem cell expansion and the design of a new generation of 3D bioreactors based on oil droplets as adherent cell culture substrates. To demonstrate the feasibility of such applications, we cultured MSCs and HPKs on oil emulsions (Fig. 4E and Supplementary Fig. 6 and 7). We found that after 7 days of culture, cells had proliferated to cover the surface of droplets. The cytoskeleton of cells spreading around such droplets clearly lined the contour of the interface (and extended from focal adhesions) and cells appeared to pack at similar densities to what would be expected from confluent cultures on TPS (Supplementary Fig. 7C). Finally, we confirmed that MSCs cultured on oil droplets can be conveniently transferred to culture dishes by simple contact of cell-seeded droplets, without detachment via trypsin or accutase treatment (Supplementary Fig. 8), or by centrifugation without enzymatic digestion (Supplementary Fig. 9). The transferred cells quickly migrated to tissue culture substrates, presented normal morphologies, assembling focal adhesions and a structured cytoskeleton comparable to that of cells transferred from cultures on TPS, and displayed high levels of viability (supplementary Fig. 9). Overall our results demonstrate the proof-of concept of stem cell culture at the surface of oil droplets, an important step for the design of a new generation of 3D bioreactors based on emulsions as microcarriers.

\section{Conclusion}

Our results demonstrate that the nanoscale mechanical properties of materials can regulate stem cell phenotype and expansion independently of bulk mechanical properties. We identify that the assembly of polymer nanosheets promoting the adsorption of ECM proteins such as fibronectin at the surface of oils is sufficient to promote cell adhesion and proliferation. We demonstrate that this process is 
mediated by classical integrin adhesion and acto-myosin contractility. Such process is surprising, considering the large body of work reporting the importance of bulk mechanics to support cell spreading. ${ }^{2-3,} 7,36,37$ However, close inspection does not reveal any major incompatibility between the currently accepted mechanisms of cell adhesion and the structure and mechanics of the liquid-liquid interfaces studied in our work. Indeed, the PLL nanosheets assembled at the surface of Novec 7500 displayed interfacial shear moduli of $3.0 \pm 0.2 \mathrm{~N} / \mathrm{m}$, despite a dry thickness of $14 \pm 2 \mathrm{~nm}$. This is remarkable as albumin nanosheets we previously reported (assembled in the presence of identical concentrations of the pro-surfactant PFBC) displayed interfacial shear moduli of $63 \pm 15 \mathrm{mN} / \mathrm{m}$ with comparable dry thicknesses. ${ }^{25}$ Extrapolated bulk shear moduli would correspond to $220 \pm 10 \mathrm{MPa}$ and 3.4 $\pm 2.3 \mathrm{MPa}$ for PLL and albumin nanosheets, respectively. This difference is proposed to arise from the difference in molecular self-assembly of PLL and albumin nanosheets: unlike albumin, which adsorbs at oil interfaces in the absence of PFBC, PLL required higher $\mathrm{pH}$ to neutralise amines from lysine side chains to react with the pro-surfactant, forming corresponding amides. This resulted in significantly higher functionalisation levels (13\%), compared to what was observed for albumin interfaces. We propose that such high functionalisation levels result in the formation of rafts of fluorinated-PLL residues forming PLL nanosheets that display high rigidity.

Therefore, the dimensions $(15-20 \mathrm{~nm})$ and mechanical properties of the nanosheets assembled would be expected to provide sufficient structure to enable cell spreading. Indeed, the thickness of focal adhesions (at which forces are transmitted from the cell cytoskeleton to the extra-cellular environment), in the range of a few tens of nanometers, ${ }^{38}$ and the thickness of actin stress fibres, are also in the range of a few tens of nanometers (for bundles), ${ }^{39}$ comparable to the dimentions of nanosheets. In addition, the mechanical properties of cytoskeletal structural elements are expected to be significantly weaker to those presently reported for PLL nanosheets (stress fibres were reported to display MPa moduli ${ }^{40}$ ). Therefore, our observations are in very good agreement with the notion that shear forces exerted by cells can be counterbalanced by the strength of the nanosheets studied. 
Hence, polymer and protein nanosheets displaying strong mechanical properties (with interfacial shear moduli in the range of a few tens of $\mathrm{mN} / \mathrm{m}$ to a few $\mathrm{N} / \mathrm{m}$ ) should be able to resist forces generated by cells during spreading. Indeed, keratinocytes spreading on stiff PLL nanosheets established a structured actin cytoskeleton, with clear stress fibres, and assembled focal adhesions, as on TPS, whereas cells adhering to soft PLL-oil interfaces did not display these structures (immunofluorescence microscopy showed instead actin rich protrusions reminiscent of the early stages of cell spreading, ${ }^{41}, 42$ Fig. 3F). MSC adhesion at liquid-liquid interfaces was found to be similarly regulated. In turn, cell adhesion to the ECM is an important process regulating the phenotype and function of many stem cells. ${ }^{26,33,43}$ However, from an engineering and biotechnology point of view, the requirement for hard, rigid substrates with strong bulk mechanical properties can be an important drawback. This is the case for the scale up of cell expansion systems ${ }^{9}$ and the fabrication of cell sheets. ${ }^{44,}{ }^{45}$ Hard rigid substrates also require enzymatic digestion for cell recovery, which can be harmful or induce changes in cell phenotype (harsh trypsin treatment decreases the colony forming efficiency of keratinocytes ${ }^{46}$ ). The use of liquid substrates directly addresses these issues and may find further application in other biotechnological platforms such as microdroplet platforms, which have been restricted by the requirements of cell adhesion. ${ }^{47}$ In addition, biomaterials and implants design will benefit from the concept that stem cell adhesion and phenotype can be engineered though the control of interface mechanics and physico-chemistry, independently of the bulk properties of associated materials.

\section{Materials and Methods}

Interfacial rheology. Rheological measurements were carried out on a hybrid rheometer (DHR-3) from TA Instruments fitted with a double wall ring (DWR) geometry and a Delrin trough with a circular channel. The double wall ring used for this geometry has a radius of $34.5 \mathrm{~mm}$ and the thickness of the Platinum-Iridium wire is $1 \mathrm{~mm}$. The diamond-shaped cross-section of the geometry's ring provides 
the capability to pin directly onto the interface between two liquids and measure the interface properties without complicated sub-phase correction. $19 \mathrm{~mL}$ of the fluorinated oil pre-mixed with surfactant were placed in the Delrin trough and the ring was lowered, ensuring contact with the surface, via an axial force procedure. The measuring position was set $500 \mu \mathrm{m}$ lower than the contact point of the ring with the oil-phase surface. Thereafter, $15 \mathrm{ml}$ of the PBS buffer were carefully syringed on top of the oil phase. Time sweeps were performed at a constant frequency of $0.1 \mathrm{~Hz}$ and a temperature of $25^{\circ} \mathrm{C}$, with a displacement of $1.010^{-3} \mathrm{rad}$ to follow the formation of the protein layers at the interface. Before and after each time sweep, frequency sweeps (with a constant displacement of $1.010^{-3} \mathrm{rad}$ ) were conducted to examine the frequency-dependant characteristics of the interface whilst amplitude sweeps (with constant frequencies of $0.1 \mathrm{~Hz}$ ) were carried out to ensure that the chosen displacement was within the linear viscoelastic region.

Generation of Liquid-Liquid Interfaces for Cell Culture: 24 well plates were plasma oxidized using a plasma coater (Diener, $100 \%$ intensity) for 10 minutes. $500 \mu \mathrm{L}$ ethanol (VWR chemicals), $10 \mu \mathrm{L}$ trimethylamine (Sigma-Aldrich) and $10 \mu \mathrm{L}$ of the desired silane (triethoxy(octyl)silane (Sigma-Aldrich) to prepare interfaces with liquid PDMS or trichloro $(1 \mathrm{H}, 1 \mathrm{H}, 2 \mathrm{H}, 2 \mathrm{H}$-perfluorooctyl) silane (SigmaAldrich) for the fluorinated oil) were added into each well. After incubating for $24 \mathrm{~h}$, the wells were washed in a sterile environment with ethanol (twice) and $\mathrm{ddH}_{2} \mathrm{O}$ (three times). $500 \mu \mathrm{L}$ fluorinated oil (Novec 7500, ACOTA) with fluorinated surfactant (2,3,4,5,6-pentafluorobenzoyl chloride, SigmaAldrich) at a final concentration $0.01 \mathrm{mg} / \mathrm{mL}$ was added in the fluorophilic 24 well plate to form the bottom liquid layer. For the deposition of poly(L-lysine) (PLL), a $20 \mu \mathrm{L}$ PLL solution (10 mg/mL) was added to PBS (at pH 7.4 or 10.5, depending on conditions), to make a final concentration of $100 \mu \mathrm{g} / \mathrm{mL}$, and incubated for $1 \mathrm{~h}$. The polymer solution was then diluted with PBS (pH 7.4) 6 times. For fibronectin adsorption, $20 \mu \mathrm{L}$ fibronectin solutions $(1 \mathrm{mg} / \mathrm{mL}$ ) were pipetted into each well (after PLL coating), making a final concentration of $10 \mu \mathrm{g} / \mathrm{mL}$, and incubated for $1 \mathrm{~h}$. The protein solution was diluted with PBS (PH 7.4) 4 times and then with growth medium twice. For the deposition of poly(L-lysine)-graft- 
poly(ethylene glycol) (PLL-PEG, Surface Solutions), interfaces were exposed to solutions of PLL-PEG $(100 \mu \mathrm{g} / \mathrm{mL})$ for $1 \mathrm{~h}$, at $\mathrm{pH} 10.5$.

\section{Acknowledgements}

We thank Khai Nguyen for assistance with interfacial rheology characterisation. We thank $\mathrm{Dr}$ Madeleine Ramstedt for XPS data and analysis. S.D.C. thanks the Institute of Bioengineering for a studentship. L.P. thanks the China Scholarship Council for a studentship (201708060335). J.E.G. and D.K. thank the Leverhulme Trust Foundation for financial support (RPG-2017-229, Grant 69241).

\section{Associated Content}

Additional materials and methods, additional nanosheets mechanics and PLL adsorption kinetics, fibronectin adsorption quantification, additional cell adhesion quantification and microscopy (spreading, FA formation, actin cytoskeleton assembly, integrin blocking), imaging of keratinocytes and MSCs spreading around droplets and MSCs transferred from droplets, statistical analysis reports. This supporting information is available free of charge at http://pubs.acs.org.

\section{References}

(1) Assoian, R. K.; Klein, E. A. Growth Control by Intracellular Tension and Extracellular Stiffness. Trends Cell Biol. 2008, 18, 347-352.

(2) Pelham, R. J.; Wang, Y.-L. Cell Locomotion and Focal Adhesions Are Regulated by Substrate Flexibility. Proc. Natl. Acad. Sci. 1997, 94, 13661-13665.

(3) Yeung, T.; Georges, P. C.; Flanagan, L. A.; Marg, B.; Ortiz, M.; Funaki, M.; Zahir, N.; Ming, W.; Weaver, V.; Janmey, P. A. Effect of Substrate Stiffness on Cell Morphology, Cytoskeletal Structure, and Adhesion. Cell Motil. Cytoskeleton 2005, 60, 24-34. 
(4) Lee-Thedieck, C.; Rauch, N.; Fiammengo, R.; Klein, G.; Spatz, J. P. Impact of Substrate Elasticity on Human Hematopoietic Stem and Progenitor Cell Adhesion and Motility. J. Cell Sci. 2012, 125, 3765-3775.

(5) Leight, J. L.; Wozniak, M. A.; Chen, S.; Lynch, M. L.; Chen, C. S. Matrix Rigidity Regulates a Switch Between TGF- $\beta 1$-Induced Apoptosis and Epithelial-Mesenchymal Transition. Mol. Biol. Cell 2012, 23, 781-791.

(6) Gilbert, P. M.; Havenstrite, K. L.; Magnusson, K. E. G.; Sacco, A.; Leonardi, N. A.; Kraft, P.; Nguyen, N. K.; Thrun, S.; Lutolf, M. P.; Blau, H. M. Substrate Elasticity Regulates Skeletal Muscle Stem Cell Self-Renewal in Culture. Science 2010, 329, 1078-1081.

(7) Engler, A. J.; Sen, S.; Sweeney, H. L.; Discher, D. E. Matrix Elasticity Directs Stem Cell Lineage Specification. Cell 2006, 126, 677-689.

(8) Saha, K.; Keung, A. J.; Irwin, E. F.; Li, Y.; Little, L.; Schaffer, D. V.; Healy, K. E. Substrate Modulus Directs Neural Stem Cell Behavior. Biophys. J. 2008, 95, 4426-4438.

(9) Chu, L.; Robinson, D. K. Industrial Choices for Protein Production by Large-Scale Cell Culture. Curr. Opin. Biotech. 2001, 12, 180-187.

(10) Warikoo, V.; Godawat, R.; Brower, K.; Jain, S.; Cummings, D.; Simons, E.; Johnson, T.; Walther, J.; Yu, M.; Wright, B.; McLarty, J.; Karey, K. P.; Hwang, C.; Zhou, W.; Riske, F.; Konstantinov, K. Integrated Continous Production of Recombinant Therapeutic Proteins. Biotech. Bioeng. 2012, 109, 3018-3029.

(11) Hacker, D. L.; De Jesus, M.; Wurm, F. M. 25 Years of Recombinant Proteins From Reactor-Grown Cells - Where Do We Go From Here? Biotech. Adv. 2009, 27, 1023-1027.

(12) Trappmann, B.; Gautrot, J. E.; Connelly, J.; Strange, D. G. T.; Li, Y.; Oyen, M. L.; Cohen Stuart, M. A.; Boehm, H.; Li, B.; Vogel, V.; Spatz, J. P.; Watt, F. M.; Huck, W. T. S. Extracellular Matrix Tethering Regulates Stem Cell Fate. Nat. Mater. 2012, 11, 642-649.

(13) Khetan, S.; Guvendiren, M.; Legant, W. R.; Cohen, D. M.; Chen, C. S.; Burdick, J. A. DegradationMediated Cellular Traction Directs Stem Cell Fate in Covalently Crosslinked Three-Dimensional Hydrogels. Nat. Mater. 2013, 12, 458-465.

(14) Huebsch, N.; Arany, P. R.; Mao, A. S.; Shvartsman, D.; Ali, O. A.; Rivera-Feliciano, J.; Mooney, D. J. Harnessing Traction-Mediated Manipulation of the Cell/Matrix Interface to Control Stem-Cell Fate. Nat. Mater. 2010, 9, 518-526.

(15) Chaudhuri, O.; Gu, L.; Darnell, M.; Klumpers, D.; Bencherif, S. A.; Weaver, J. C.; Huebsch, N.; Mooney, D. J. Substrate Stress Relaxation Regulates Cell Spreading. Nat. Commun. 2015, 6, 6364.

(16) Chaudhuri, O.; Gu, L.; Klumpers, D.; Darnell, M.; Bencherif, S. A.; Weaver, J. C.; Huebsch, N.; Lee, H.-P.; Lippens, E.; Duda, G. N.; Mooney, D. J. Hydrogels With Tunable Stress Relaxation Regulate Stem Cell Fate and Activity. Nat. Mater. 2016, 15, 326-334.

(17) Attwood, S. J.; Cortes, E.; Haining, A. W. M.; Robinson, B.; Li, D.; Gautrot, J. E.; del Rio Hernandez, A. Adhesive Ligand Tether Length Affects the Size and Length of Focal Adhesions and Influences Cell Spreading and Attachment. Sci. Rep. 2016, 6, 34334.

(18) Moore, S. W.; Roca-Cusachs, P.; Sheetz, M. P. Stretchy Proteins on Stretchy Substrates: the Important Elements of Integrin-Mediated Rigidity Sensing. Dev. Cell 2010, 19, 194-206.

(19) Di Cio, S.; Gautrot, J. E. Cell Sensing of Physical Properties at the Nanoscale: Mechanisms and Control of Cell Adhesion and Phenotype. Acta Biomater 2016, 30, 26-48.

(20) Giaever, I.; Keese, C. R. Behavior of Cells at Fluid Interfaces. Proc. Natl. Acad. Sci. 1983, 80, 219222.

(21) Keese, C. R.; Giaever, I. Cell Growth on Liquid Microcarriers. Science 1983, 219, 1448-1449.

(22) Keese, C. R.; Giaever, I. Cell Growth on Liquid Interfaces: Role of Surface Active Compounds. Proc. Natl. Acad. Sci. 1983, 80, 5622-5626.

(23) Minami, K.; Mori, T.; Nakanishi, W.; Shigi, N.; Nakanishi, J.; Hill, J. P.; Komiyama, M.; Ariga, K. Suppression of Myogenic Differentiation of Mammalian Cells Caused by Fluidity of a Liquid-Liquid Interface. Appl. Mater. Interfaces 2017, 9, 30553-30560. 
(24) Kong, D.; Nguyen, K. D. Q.; Megone, W.; Peng, L.; Gautrot, J. E. The culture of HaCaT Cells on Liquid Substrates Is Mediated by a Mechanically Strong Liquid-Liquid Interface. Faraday Discuss. 2017, 204, 367-381.

(25) Kong, D.; Megone, W.; Nguyen, K. D. Q.; di Cio, S.; Ramstedt, M.; Gautrot, J. E. Protein Nanosheet Mechanics Controls Cell Adhesion and Expansion on Low-Viscosity Liquids. Nano Lett. 2018, 18, 1946-1951.

(26) Watt, F. M. Role of Integrins in Regulating Epidermal Adhesion, Growth and Differentiation. EMBO J. 2002, 21, 3919-3926.

(27) Guilak, F.; Cohen, D. M.; Estes, B. T.; Gimble, J. M.; Liedtke, W.; Chen, C. S. Control of Stem Cell Fate by Physical Interactions With the Extracellular Matrix. Cell Stem Cell 2009, 5, 17-26.

(28) Fuller, G. G.; Vermant, J. Complex Fluid-Fluid Interfaces: Rheology and Structure. Annu. Rev. Chem. Biomol. Eng. 2012, 3, 519-543.

(29) Freer, E. M.; Yim, K. S.; Fuller, G. G.; Radke, C. J. Interfacial Rheology of Globular and Flexible Proteins at the Hexadecane/Water Interface: Comparison of Shear and Dilatation Deformation. J. Phys. Chem. B 2004, 108, 3835-3844.

(30) Karbaschi, M.; Lotfi, M.; Kragel, J.; Javadi, A.; Bastani, D.; Miller, R. Rheology of Interfacial Layers. Curr. Opin. Coll. Interf. Sci. 2014, 19, 514-519.

(31) Levy, L.; Broad, S.; Diekmann, D.; Evans, R. D.; Watt, F. M. $\beta 1$ Integrins Regulate Keratinocyte Adhesion and Differentiation by Distinct Mechanisms. Mol. Biol. Cell 2000, 11, 453-466.

(32) Novak, P.; Li, C.; Shevchuk, A. I.; Stepanyan, R.; Caldwell, M.; Hughes, S.; Smart, T. G.; Gorelik, J.; Ostanin, V. P.; Lab, M. J.; Moss, G. W. J.; Frolenkov, G. I.; Klenerman, D.; Korchev, Y. E. Nanoscale Live-Cell Imaging Using Hopping Probe Ion Conductance Microscopy. Nat. Mater. 2009, 6, 279281.

(33) Discher, D. E.; Mooney, D. J.; Zandstra, P. W. Growth Factors, Matrices, and Forces Combine and Control Stem Cells. Science 2009, 324, 1673-1677.

(34) Bennett, M.; Cantini, M.; Reboud, J.; Cooper, J. M.; Roca-Cusachs, P.; Salmeron-Sanchez, M. Molecular Clutch Drives Cell Response to Surface Viscosity. Proc. Natl. Acad. Sci. 2018, 115, 11921197.

(35) Janes, S. M.; Ofstad, T. A.; Campbell, D. H.; Eddaoudi, A.; Warnes, G.; Davies, D.; Watt, F. M. Pi3Kinase Dependent Activation of Apoptotic Machinery Occurs on Commitment of Epidermal Keratinocytes to Terminal Differentiation. Cell Res. 2009, 19, 328-339.

(36) Discher, D. E.; Janmey, P.; Wang, Y.-L. Tissue Cells Feel and Respond to the Stiffness of Their Substrate. Science 2005, 310, 1139-1143.

(37) Georges, P. C.; Janmey, P. A. Cell Type-Specific Response to Growth on Soft Materials. J. Appl. Physiol. 2005, 98, 1547-1553.

(38) Kanchanawong, P.; Shtengel, G.; Pasapera, A. M.; Ramko, E. B.; Davidson, M. W.; Hess, H. F.; Waterman, C. M. Nanoscale Architecture of Integrin-Based Cell Adhesions. Nature 2010, 468, 580-584.

(39) Blanchoin, L.; Boujemaa-Paterski, R.; Sykes, C.; Plastino, J. Actin Dynamics, Architecture, and Mechanics in Cell Motility. Physiol. Rev. 2014, 94, 235-263.

(40) Deguchi, S.; Ohashi, T.; Sato, M. Tensile Properties of Single Stress Fibers Isolated From Cultured Vascular Smooth Muscle Cells. J. Biomech. 2006, 39, 2603-2610.

(41) Parsons, J. T.; Horwitz, A. R.; Schwartz, M. A. Cell Adhesion: Integrating Cytoskeletal Dynamics and Cellular Tension. Nat. Rev. 2010, 11, 633-643.

(42) Gardel, M. L.; Sabass, B.; Ji, L.; Danuser, G.; Schwarz, U. S.; Waterman, C. M. Traction Stress in Focal Adhesions Correlates Biphasically with Actin Retrograde Flow Speed. J. Cell Biol. 2008, 183, 999-1005.

(43) Fujiwara, H.; Ferreira, M.; Donati, G.; Marciano, D. K.; Linton, J. M.; Sato, Y.; Hartner, A.; Sekiguchi, K.; Reichardt, L. F.; Watt, F. M. The Basement Membrane of Hair Follicle Stem Cells is a Muscle Cell Niche. Cell 2011, 144, 577-589. 
(44) Elloumi-Hannachi, I.; Yamato, M.; Okano, T. Cell Sheet Engineering: A Unique Nanotechnology for Scaffold-Free Tissue Reconstruction With Clinical Applications in Regenerative Medicine. J. Internal Med. 2010, 267, 54-70.

(45) Matsuda, N.; Shimizu, T.; Yamato, M.; Okano, T. Tissue Engineering Based on Cell Sheet Technology. Adv. Mater. 2007, 19, 3089-3099.

(46) Jones, P. H.; Watt, F. M. Separation of Human Epidermal Stem Cells from Transit Amplifying Cells on the Basis of Differences in Integrin Function and Expression. Cell 1993, 73, 713-724.

(47) Huebner, A.; Sharma, S.; Srisa-Art, M.; Hollfelder, F.; Edel, J. B.; deMello, A. J. Microdroplets: A Sea of Applications? Lab Chip 2008, 8, 1244-1254. 

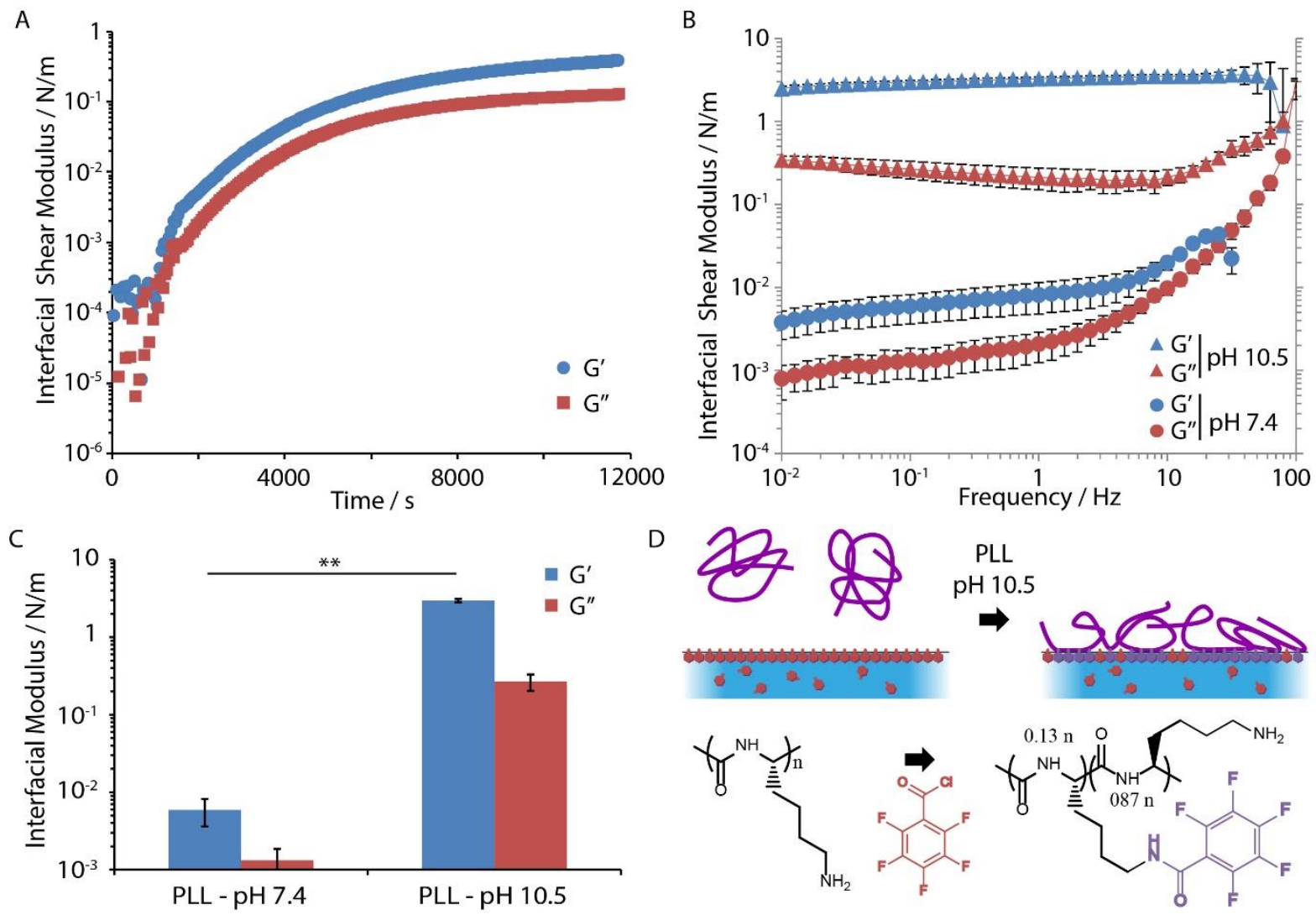

Figure 1. PLL nanosheets assembled at liquid-liquid interfaces display strong mechanical properties.

A. Evolution of the interfacial shear moduli of Novec 7500/PBS interfaces during the assembly of PLL $(100 \mu \mathrm{g} / \mathrm{mL})$ in the presence of the pro-surfactant PFBC $(10 \mu \mathrm{g} / \mathrm{mL}$ in the oil); time sweeps recorded at oscillation frequencies of $0.1 \mathrm{~Hz}$ and oscillating amplitude of $10^{-3} \mathrm{rad}$. B and C. Frequency sweep experiments (oscillating amplitude of $10^{-4} \mathrm{rad}$ ) carried out for the characterisation of PLL-nanosheets assembly at interfaces generated at a concentration of PFBC of $10 \mu \mathrm{g} / \mathrm{mL}$, with a PLL concentration of $100 \mu \mathrm{g} / \mathrm{mL}$, at $\mathrm{pH} 7.4$ and 10.5. D. Schematic representation of the proposed molecular assembly of PLL at oil-water interfaces, assisted by the pro-surfactant PFBC. 
A
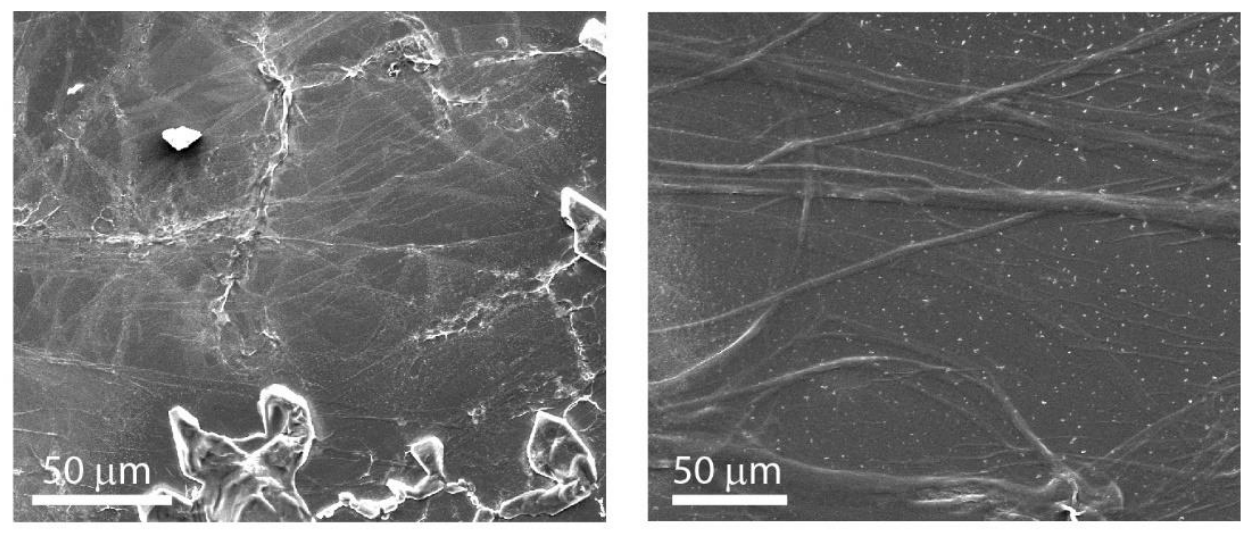

B

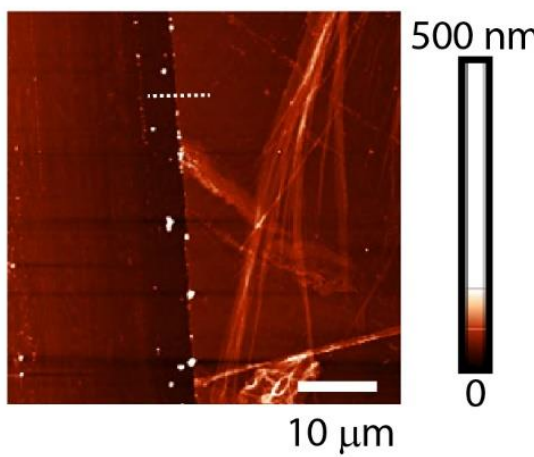

C

C 1S

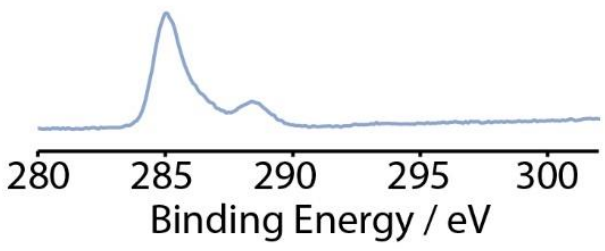

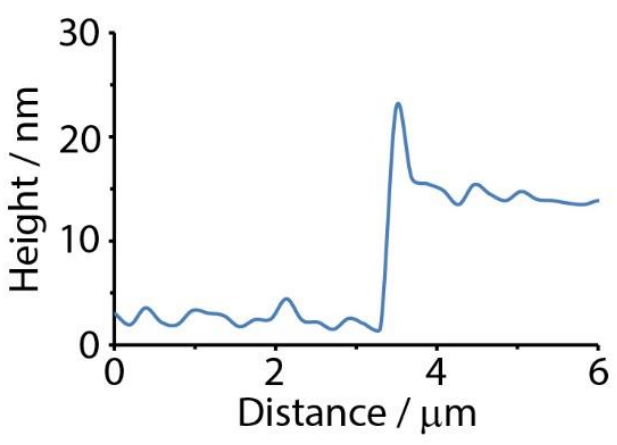

F 1S

Figure 2. PLL adsorption at the surface of fluorinated oils, assisted by coupling with the prosurfactant PFBC, forms nanosheets. A. SEM images of PLL nanosheets assembled at Novec 75000/PBS interfaces $([P F B C]=0.01 \mathrm{mg} / \mathrm{mL},[P L L]=100 \mu \mathrm{g} / \mathrm{mL})$, deposited onto silicon substrates. $B$. AFM characterisation (height image, profile and quantification of thickness) of corresponding PLL nanosheets. Error bars are s.e.m.; $n>50$. C and D. XPS spectra (C, C 1S; D, F 1S) obtained for corresponding PLL nanosheets. 


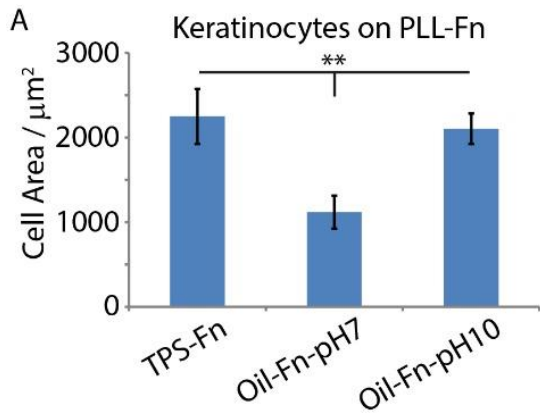

B

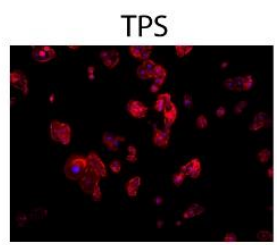

PLL - pH 7.4

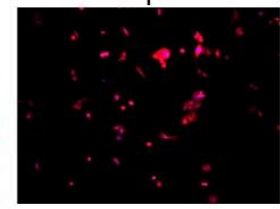

PLL - pH 10.5

C HaCaTs on PLL + inhibitors

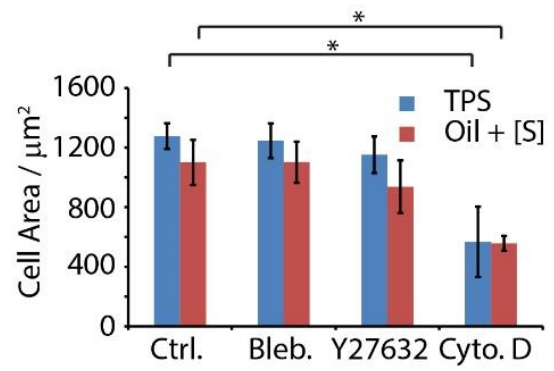

$\mathrm{F}$
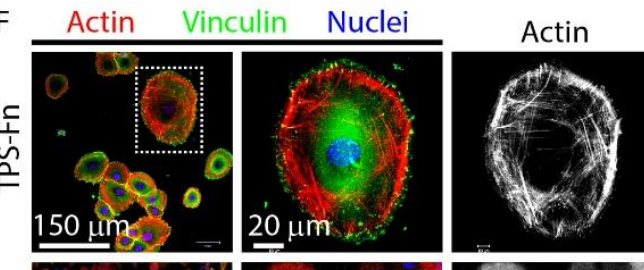

Vinculin
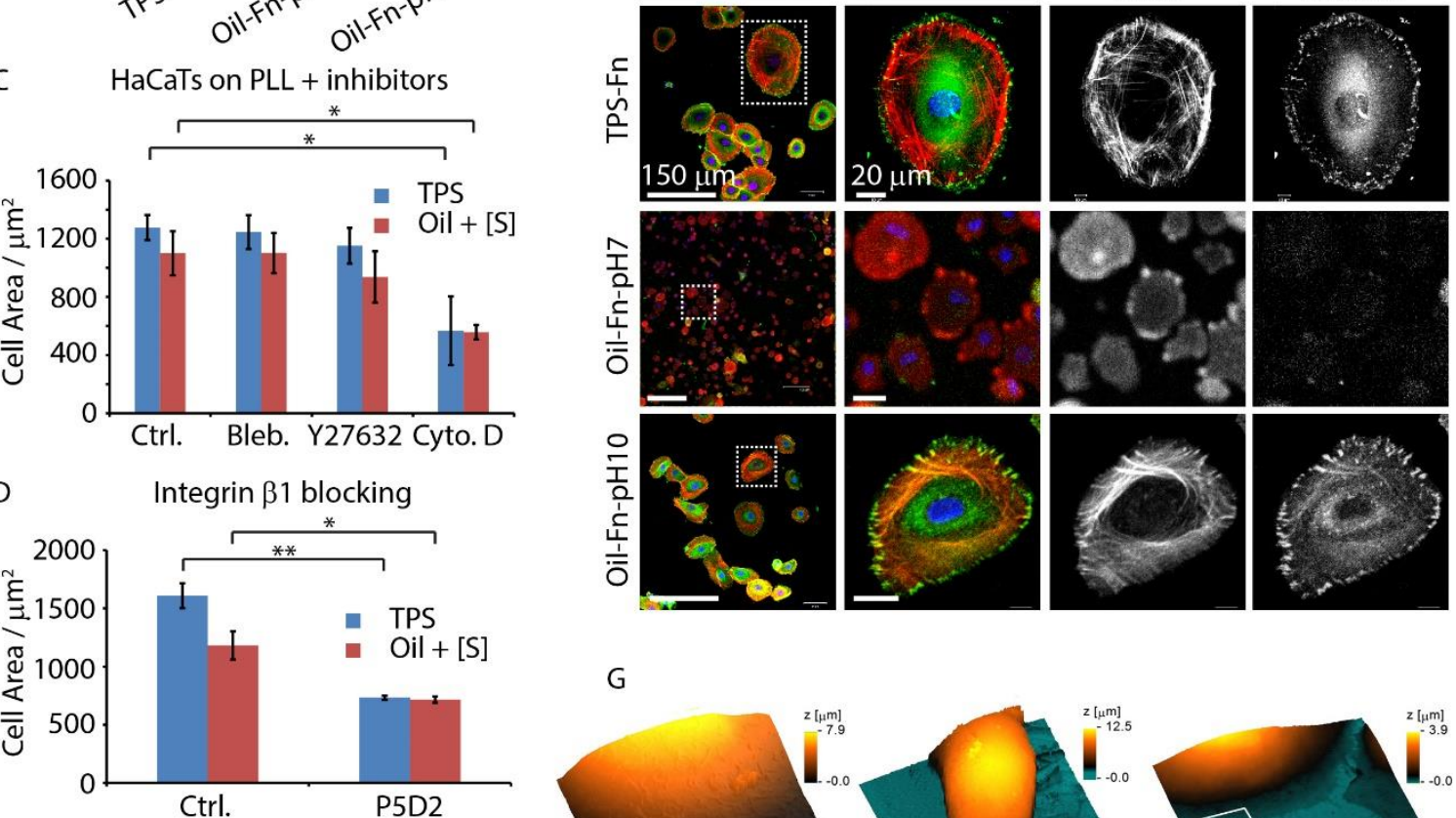

Ctrl.

P5D2

E

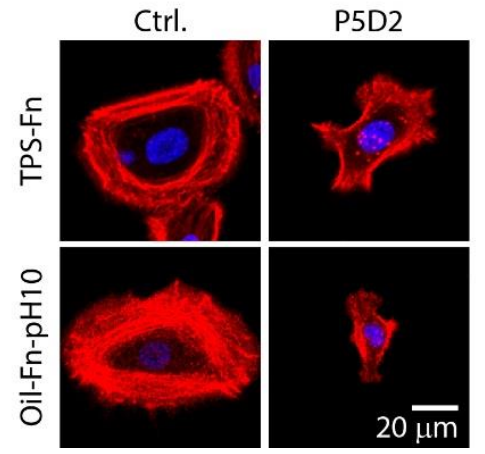

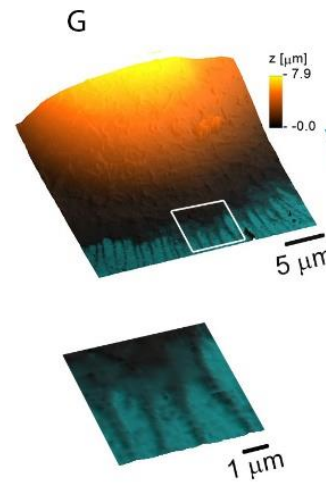

TPS-Fn
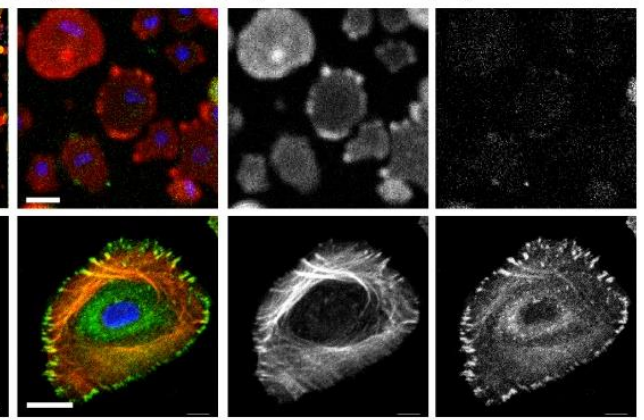
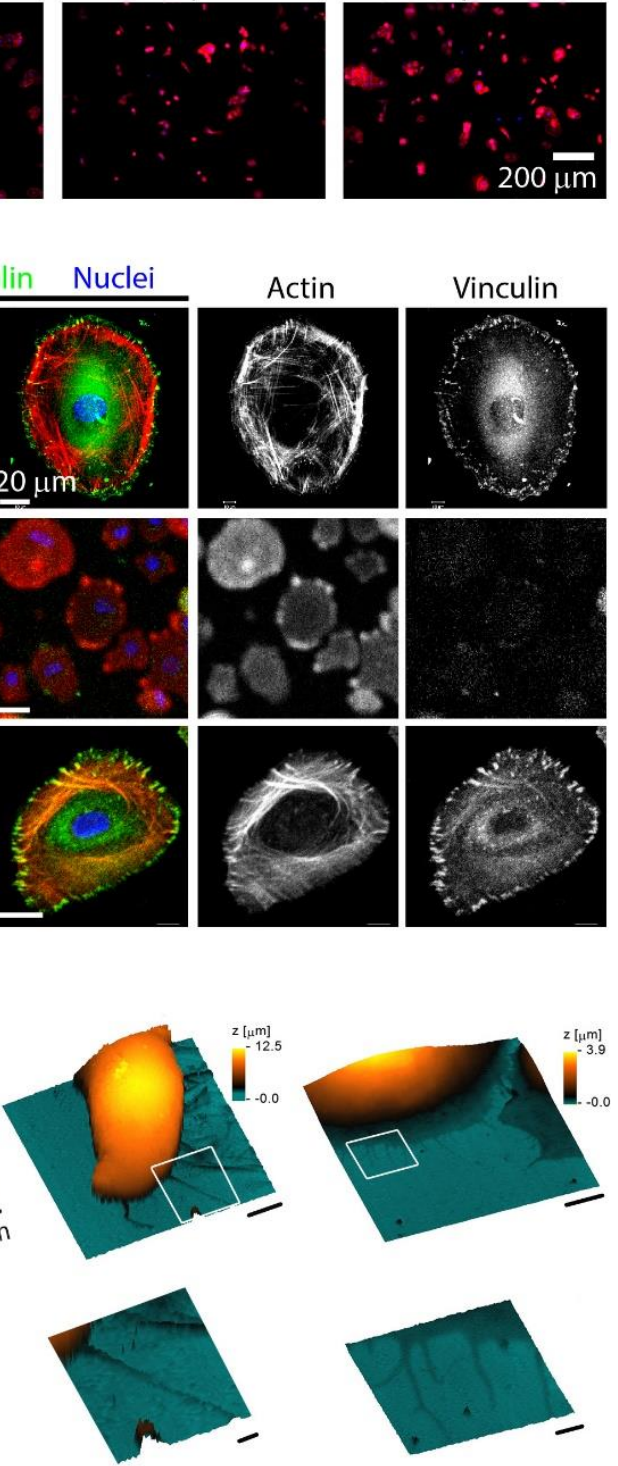

Oil-Fn-pH7

Oil-Fn-pH10

Figure 3. Cell spreading at liquid-liquid interfaces is mediated by integrin adhesion and regulated by acto-myosin contractility. A. Human primary keratinocyte (HPK) spreading (after $24 \mathrm{~h}$ ) on TPS and PLL-fibronectin functionalised oil interfaces (functionalised with PLL at pH 7.4 or 10.5, then fibronectin at neutral pH). B. Corresponding fluorescence images (Red, actin; Blue, DAPI). C. HaCaT cells spreading on BSA interfaces (TPS and Novec $7500+$ PFBC) is modulated by the action of acto-myosin inhibitors (myosin inhibitor blebbistatin, $10 \mu \mathrm{M}$; ROCK inhibitor Y27632, $10 \mu \mathrm{M}$; actin polymerisation inhibitor Cytochalasin D, $1 \mu \mathrm{M})$. Cell areas determined from actin stainings (phalloidin). D. Blocking of $\beta_{1}$ integrins in HPK cells spreading on PLL-FN interfaces (TPS and Novec 7500 + PFBC; blocking with mouse anti- $\beta_{1}$ integrin antibody P5D2, 1:50, $20 \mu \mathrm{g} / \mathrm{mL}$ ). PLL deposition was carried out in $\mathrm{pH} 10.5$ PBS at the surface of fluorinated oil. Cell areas determined from actin stainings (phalloidin). E. Corresponding fluorescence microscopy images (blue, DAPI; red, phalloidin). F. Confocal microscopy images of HPKs spreading (after $24 \mathrm{~h}$ ) on TPS and PLL-functionalised oil interfaces. Zooms correspond to the dotted 
boxes. G. SICM imaging in hopping mode of HPKs spreading (after $24 \mathrm{~h}$ ) on TPS and PLL-functionalised oil interfaces. Zooms correspond to the dotted boxes. Error bars are s.e.m.; n=3.

A

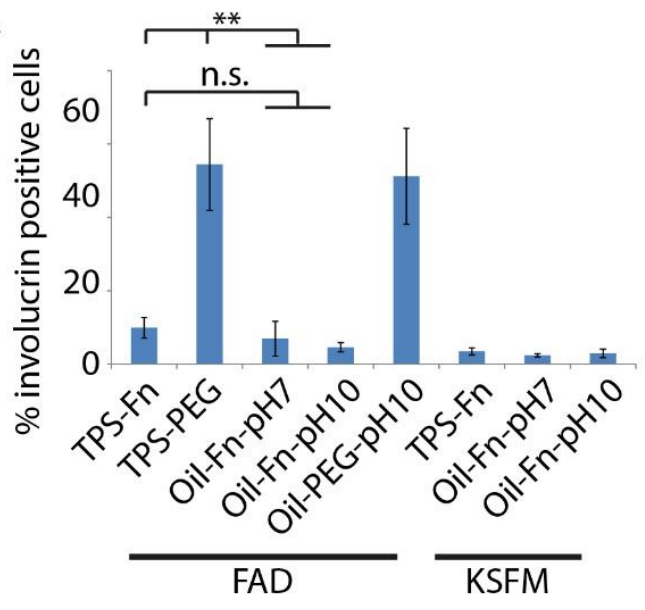

Nuclei

Involucrin Actin $200 \mu \mathrm{m}$
B
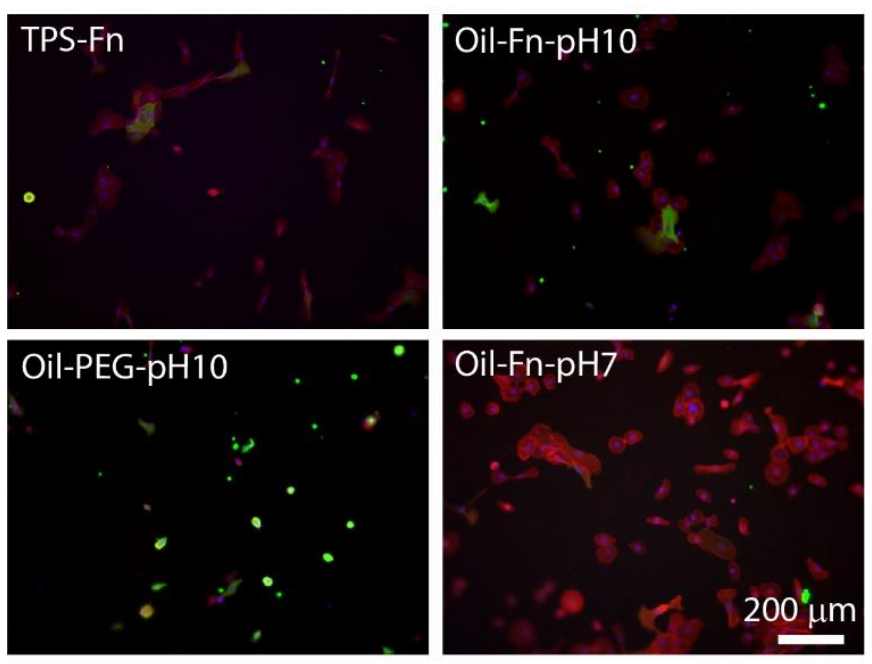

C

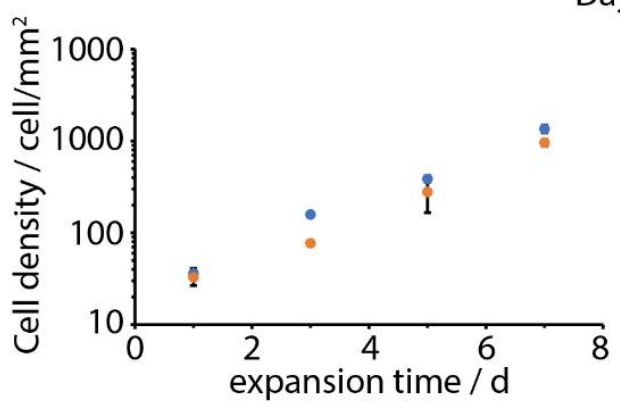

Day 5

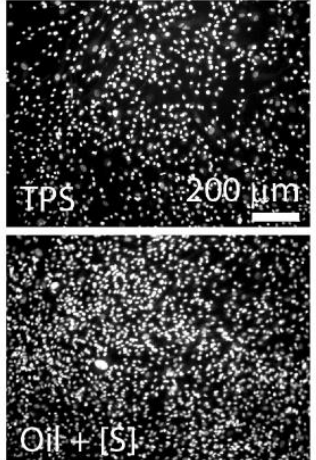

$\mathrm{E}$
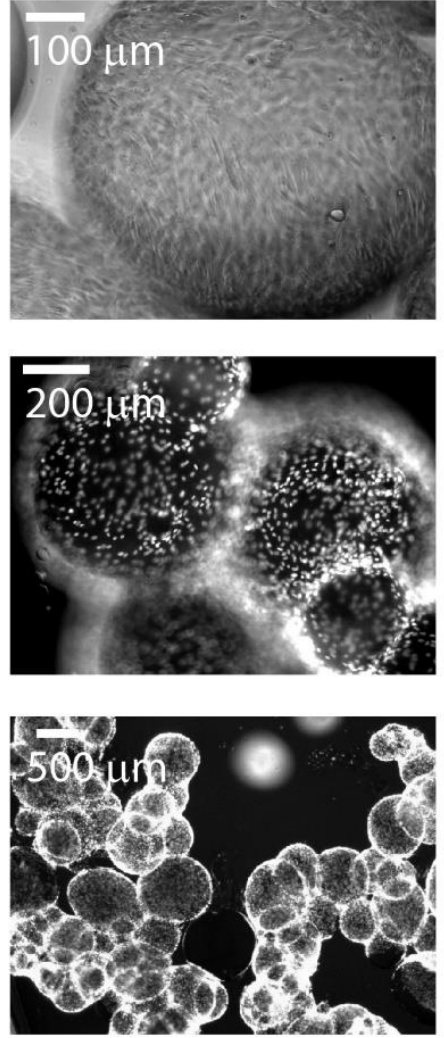

Figure 4. Stem cell differentiation and expansion at liquid-liquid interfaces. A. HPK differentiation on solid (TPS functionalised with fibronectin, Fn, or PLL-PEG) and liquid interfaces (Novec $7500+0.01$ $\mathrm{mg} / \mathrm{mL}$ PFBC; oil-Fn-pH7, PLL at pH 7.4 then Fn; oil-Fn-pH10, PLL at pH 10.5 then Fn; oil-PEG-pH10, PLL-PEG at pH 10.5) in differentiation medium (FAD) and basal medium (KSFM). Error bars are s.e.m.; $n=3$. B. Fluorescence microscopy images (red, actin; green, involucrin; blue, DAPI) corresponding to some of these conditions. C. MSCs proliferation profile on fluorinated oil interfaces functionalised with 
PLL-Fibronectin (Fn) at pH 10.5 (PLL, $100 \mu \mathrm{g} / \mathrm{mL}$; Fn, 10 g/mL; red, TPS; blue, Novec $7500+0.00125$ $\mathrm{mg} / \mathrm{mL}$ PFBC). Error bars are s.e.m.; $n=3$. Images are corresponding nuclear stainings at Day 5 (Hoechst, scale bars are $200 \mu \mathrm{m}$ ). D. Confocal microscopy images of MSCs spreading (after $24 \mathrm{~h}$ ) on TPS and PLL-functionalised oil. Zooms correspond to the dotted boxes. E. Micrographs of MSCs cultured on emulsions for 7 days in growth medium (top bright field; middle and bottom, epifluorescence images of Hoechst stainings). 